\title{
A METHOD FOR CALCULATING LINKAGE VALUES ${ }^{1}$
}

\author{
HUGO W. ALBERTS \\ Department of Agronomy, University of Illinois, Urbana, Illinois \\ Received August 7, 1925
}

TABLE OF CONTENTS

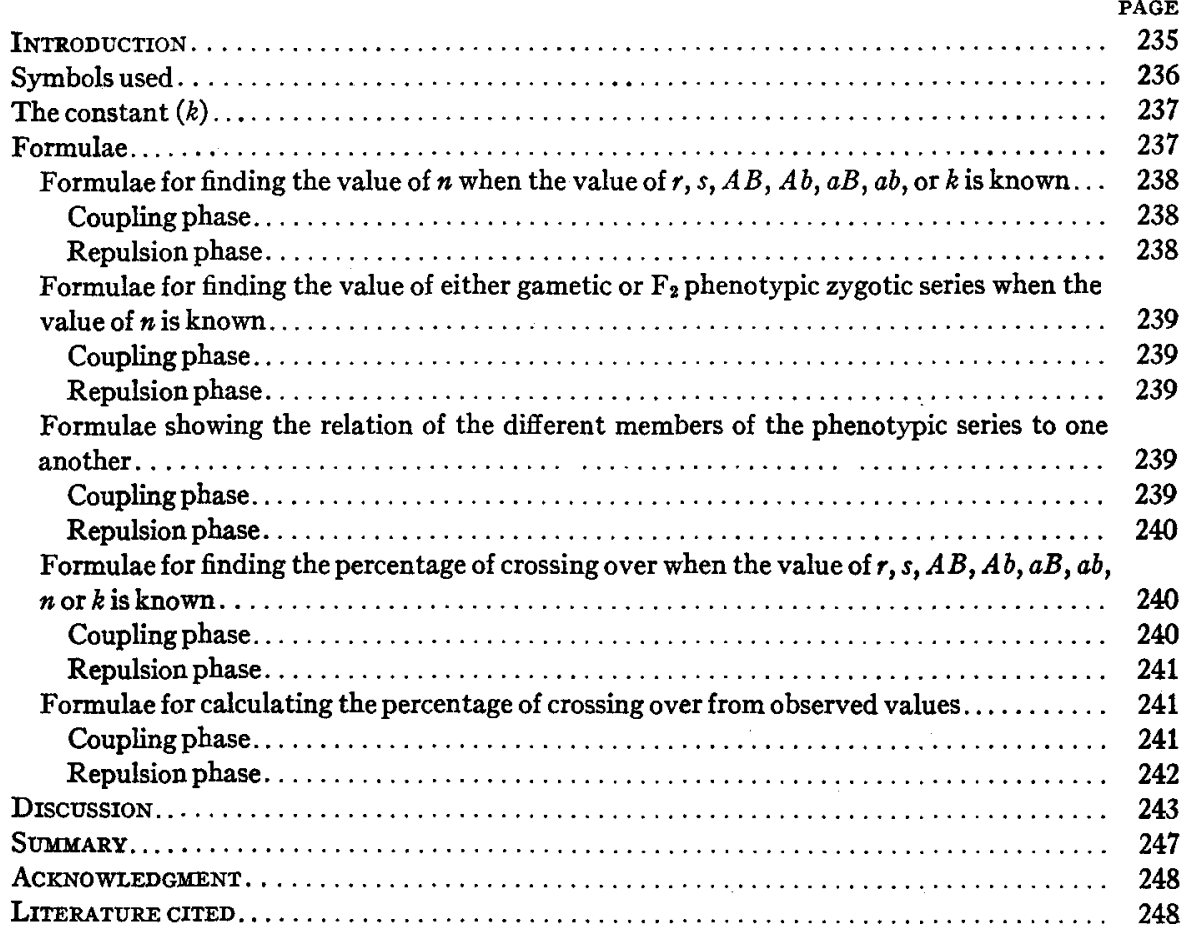

INTRODUCTION

The calculation of linkage values in many plants is confined to $F_{2}$ distributions, due to difficulties in making backcrosses. EMERSON (1916), HALDANE (1919), and other authors have proposed methods by which the gametic ratio can be obtained from the observed phenotypic zygotic proportions and from this ratio the percentage of crossing over can be calculated. WOODWORTH (1916), ColLINS (1924), BRUNSON (1924) and

${ }^{1}$ Contribution from the Division of Plant Breeding, Department of Agronomy, UnIversity of Illinors. Published with the approval of the Director of the Station.

GenETICS 11: 235 My 1926 
others have modified or extended Emerson's method for use in special cases, involving duplicate, complementary or supplementary factors.

A study of the theoretical gametic ratios with their corresponding phenotypic zygotic ratios in a dihybrid $\mathbf{F}_{2}$ distribution (table 1) has revealed some interesting relationships. Some of these relationships have already been worked out by the authors cited above, but most of those contained in this paper have not been published so far as I know. It seems desirable, therefore, to present these relationships together with additional formulae for measuring linkage intensities.

\section{TABLE 1}

Giving a number of different gametic series together with their corresponding theoretical $\mathrm{F}_{2}$ phenotypic series.

\begin{tabular}{|c|c|c|c|c|c|c|c|c|c|}
\hline \multicolumn{4}{|c|}{ GAMETIC SERIES } & \multicolumn{4}{|c|}{$F_{2}$ PHENOTXPIC SERIES } & \multirow{2}{*}{$\operatorname{TOTAL}(n)$} & \multirow{2}{*}{ CONSTANT $(k)$} \\
\hline$r$ & $s$ & $s$ & $r$ & $A B$ & $A b$ & $a B$ & $a b$ & & \\
\hline 10 & 1 & 1 & 10 & 342 & 21 & 21 & 100 & 484 & 14.611 \\
\hline 9 & 1 & 1 & 9 & 281 & 19 & 19 & 81 & 400 & 14.48 \\
\hline 8 & 1 & 1 & 8 & 226 & 17 & 17 & 64 & 324 & 14.320 \\
\hline 7 & 1 & 1 & 7 & 177 & 15 & 15 & 49 & 256 & 14.125 \\
\hline 6 & 1 & 1 & 6 & 134 & 13 & 13 & 36 & 196 & 13.877 \\
\hline 5 & 1 & 1 & 5 & 97 & 11 & 11 & 25 & 144 & 13.555 \\
\hline 4 & 1 & 1 & 4 & 66 & 9 & 9 & 16 & 100 & 13.12 \\
\hline 3 & 1 & 1 & 3 & 41 & 7 & 7 & 9 & 64 & 12.5 \\
\hline 2 & 1 & 1 & 2 & 22 & 5 & 5 & 4 & 36 & 11.55 .5 \\
\hline 1 & 1 & 1 & 1 & 9 & 3 & 3 & 1 & 16 & 10 \\
\hline 1 & 2 & 2 & 1 & 19 & 8 & 8 & 1 & 36 & 8.888 \\
\hline 1 & 3 & 3 & 1 & 33 & 15 & 15 & 1 & 64 & 8.5 \\
\hline 1 & 4 & 4 & 1 & 51 & 24 & 24 & 1 & 100 & 8.32 \\
\hline 1 & 5 & 5 & 1 & 73 & 35 & 35 & 1 & 144 & 8.222 \\
\hline 1 & 6 & 6 & 1 & 99 & 48 & 48 & 1 & 196 & 8.163 \\
\hline 1 & 7 & 7 & 1 & 129 & 63 & 63 & 1 & 256 & 8.125 \\
\hline 1 & 8 & 8 & 1 & 16.3 & 80 & 80 & 1 & 324 & 8.098 \\
\hline 1 & 9 & 9 & 1 & 201 & 99 & 99 & 1 & 400 & 8.08 \\
\hline 1 & 10 & 10 & 1 & 243 & 120 & 120 & 1 & 484 & 8.066 \\
\hline
\end{tabular}

SYMBOLS USED

The following symbols will be used:

$r$, the first and fourth member of the gametic series $(r=1$ in the repulsion phase).

$s$, the second and third member of the gametic series $(s=1$ in the coupling phase).

$A B, A b, a B$ and $a b$, theoretical values of the members of the $\mathrm{F}_{2}$ phenotypic zygotic series. 
$A B_{0}, A b_{0}, a B_{0}$ and $a b_{0}$ observed values of the members of the $\mathrm{F}_{2}$ phenotypic zygotic series.

$n$, the sum of $A B, A b, a B$ and $a b$.

$n_{0}$, the sum of $A B_{0}, A b_{0}, a B_{0}$ and $a b_{0}$.

$k$, constant for a given series obtained by dividing the sum of $A B$ and $a b$ by $n$ (or by dividing the sum of $A B_{0}$ and $a b_{0}$ by $n_{0}$ ) and multiplying the quotient by 16, (formulae 1 and 2).

$E_{0}$, the sum of $A B_{0}$ and $a b_{0}$.

$M_{0}$, the sum of $A b_{0}$ and $a B_{0}$.

$c$, coefficient obtained by dividing $n_{0}$ by $n$.

\section{THE CONSTANT $(k)$}

As is shown in table 1 , the value of the constant $k$ is dependent on the relationship existing between the genetic factors concerned. If there is independent inheritance, $k$ has a value of 10 ; if $A$ and $B$ are coupled, the value of $k$ varies from 10 to 16 , depending on the strength of the coupling; and if $A$ and $b$ are linked (repulsion phase), $k$ varies from 10 to 8 depending on the strength of the linkage. If the linkage in the coupling phase is complete, $k$ has a value of 16 , but in case of complete linkage in the repulsion phase, $k$ has a value of 8 .

Figure 1 shows that as the value of $r$ (coupling phase) increases, the value of the constant $k$ also increases, approaching 16 as a limit. Figure 2 shows that as the value of $s$ (repulsion phase) increases, the value of the constant $k$ decreases, approaching 8 as a limit. Table 2 shows that when large values for $r$ and $s$ are assumed, the constant $k$ approaches 16 in case of coupling and 8 in case of repulsion. With such high values for $r$ and $s$ the linkage is practically complete.

TABLE 2

Showing the value of $k$ when the values of $r$ (coupling) and $s$ (repulsion) are extremely high.

\begin{tabular}{|c|c|c|c|c|c|}
\hline \multicolumn{4}{|c|}{ GAMETIC SERIES } & \multirow{2}{*}{ TOTAL $(n)$} & \multirow{2}{*}{ CONSTANT $(k)$} \\
\hline$r$ & $s$ & $s$ & $r$ & & \\
\hline 3199 & 1 & 1 & 3199 & $40,960,000$ & 15.99500078125 \\
\hline 1 & 3199 & 3199 & 1 & $40,960,000$ & 8.00000078125 \\
\hline
\end{tabular}

FORMULAE

The formulae given below are applicable when deviations from a dihybrid Mendelian $F_{2}$ distribution are caused by linkage. When such disturbances as differential viability of gametes or failure of many seeds of certain types to germinate are operating, the results obtained by the application of the formulae to observed values will be misleading. The 
following formula shows the method of obtaining the value of $k$ from the theoretical $\mathrm{F}_{2}$ phenotypic series:

$$
k=\frac{16(A B+a b)}{n}
$$

The general formula also holds for obtaining the value of $k$ from observed $\mathrm{F}_{2}$ phenotypic ratios:

$$
k=\frac{16\left(A B_{0}+a b_{0}\right)}{n_{0}}
$$

Most of the formulae that follow indicate the relationship of the members of theoretical series. Several formulae are given to show the relationship when observed values are used. All formulae which are applicable to observed values are applicable also to theoretical values, but formulae which are applicable to theoretical values are applicable to observed values only when the value of $c$ is unity. The theoretical values can be calculated from the observed values by means of the constant $k$.

Formulae for finding the value of $n$ when the value of $r, s, A B, A b, a B$, $a b$, or $k$ is known

Coupling phase

$$
n=4(r+1)^{2}
$$

From formulae (15) and (17) the following value for $A B$ is derived,

Hence,

$$
A B=\frac{n}{2}+\left(\frac{\sqrt{n}}{2}-1\right)^{2}
$$

$$
\begin{aligned}
n & =\left[\frac{2 \sqrt{3 A B}-2+2}{3}\right]^{2} \\
n & =(A b+1)^{2} \text { or }(a B+1)^{2} \\
n & =[2(\sqrt{a b}+1)]^{2} \\
n & =\left[\frac{4(\sqrt{2 k}-\overline{16}+4)}{16-k}\right]^{2} \\
& \text { Repulsion phase } \\
n & =4(s+1)^{2} \\
n & =2(A B-1) \\
n & =4(A b+1) \\
n & =\frac{32}{k-8}
\end{aligned}
$$


Formulae for finding the value of any member of either gametic or $F_{2}$ phenotypic series when the value of $n$ is known

Coupling phase

$$
r=\frac{\sqrt{n}}{2}-1
$$

$A B=\frac{3 n-4(\sqrt{n}-1)}{4}$ (derived from formula 5$)$

$$
A B=\frac{n}{2}+a b
$$

$$
\begin{aligned}
A b \text { or } a B & =\sqrt{n}-1 \\
a b & =\left[\frac{\sqrt{n}}{2}-1\right]^{2} \\
k & =\frac{16\left[(\sqrt{n}-1)^{2}+1\right]}{n}
\end{aligned}
$$

Repulsion phase

$$
\begin{aligned}
s & =\frac{\sqrt{n}}{2}-1 \\
A B & =\frac{n}{2}+1
\end{aligned}
$$

$$
A b \text { or } a B=\frac{n}{4}-1
$$

$$
k=\frac{8(n+4)}{n}
$$

Formulae showing the relation of the different members of the phenotypic series to one another

\section{Coupling phase}

Substituting in formula 4 , the value' of $n$ obtained in formula 6 , we have

which reduces to

$$
A B=\frac{(A b+1)^{2}}{2}+\left[\frac{A b+1}{2}-1\right]^{2}
$$

$$
A B=\frac{3(A b)^{2}+2 A b+3}{4}
$$


Substituting in formula 4 , the value of $n$ obtained in formula 7 , we have

which reduces to

$$
A B=\frac{[2(\sqrt{a b}+1)]^{2}}{2}+\left[\frac{2 \sqrt{a b}+2}{2}-1\right]^{2}
$$

$$
\begin{aligned}
& A B=3 a b+4 \sqrt{a b}+2 \\
& A b=\frac{2 \sqrt{3 \overline{A B}-2}-1}{3}-(\text { derived from formula } 23)
\end{aligned}
$$

Substituting in formula 16 , the value of $n$ obtained in formula 7 , we have

$$
A b=2 \sqrt{a b}+1
$$

Substituting in formula 17 , the value of $n$ obtained in formula 5 , we have

which reduces to

$$
a b=\left[\frac{\sqrt{3 A \bar{B}-2}+1}{3}-1\right]^{2}
$$

$$
a b=\frac{3 A B-4 \sqrt{3 A \bar{B}} \overline{-2}+2}{9}
$$

Substituting in formula 17 , the value of $n$ obtained in formula 6 , we have

which reduces to

$$
a b=\left[\frac{A b+1}{2}-1\right]^{2}
$$

$$
\begin{aligned}
& a b= {\left[\frac{A b-1}{2}\right]^{2} } \\
& \text { Repulsion phase } \\
& A B=2 A b+3
\end{aligned}
$$

Formulae for finding the percentage of crossing over when the value of $r, s, A B$, $A b, a B, a b, n$ or $k$ is known

$$
\begin{gathered}
\text { Coupling phase } \\
\text { Percentage of crossing over }=100\left[\frac{1}{r+1}\right]
\end{gathered}
$$

From formulae 3 and 4 we derive

$$
A B=\frac{4(r+1)^{2}}{2}+\left[\frac{2(r+1)}{2}-1\right]^{2}
$$

From this equation we obtain

$$
r=\frac{\sqrt{3 A B-2}-2}{3}
$$


Substituting in formula 30, the value of $r$ obtained in formula 31, we have

$$
\begin{aligned}
& \text { Percentage of crossing over }=100\left[\frac{3}{\sqrt{3 A B-2}+1}\right] \\
& \qquad r=\frac{A b-1}{2} \text { (derived from formulae } 3 \text { and } 6 \text { ) }
\end{aligned}
$$

From formulae 30 and 33 we derive

$$
\begin{aligned}
& \text { Percentage of crossing over }=100\left[\frac{2}{A b+1}-\right] \\
& \qquad r=\sqrt{a b} \quad \text { (derived from formulae } 3 \text { and } 7)
\end{aligned}
$$

From formulae 30 and 35 we derive

$$
\begin{aligned}
& \text { Percentage of crossing over }=100\left[\frac{1}{\sqrt{a b}+1}\right] \\
& \text { Percentage of crossing over }=100\left[\frac{2}{\sqrt{ } \frac{1}{n}}\right] \text { (derived from }
\end{aligned}
$$

formulae 3 and 30)

From formula 8 we derive

$$
r+1=\frac{2(\sqrt{2(\bar{k}-\overline{8})}+4)}{16-k}
$$

Hence, Percentage of crossing over $=100\left[\frac{16-k}{2 \sqrt{2(k-8)}+8}\right]$

Repulsion phase

$$
\begin{aligned}
& \text { Percentage crossing over }=100\left[\frac{2}{\sqrt{2(A B-1)}}\right] \\
& \text { Percentage crossing over }=100\left[\frac{1}{\sqrt{A b+1}}\right] \\
& \text { Percentage crossing over }=100\left[\frac{2}{\sqrt{n}}\right] \\
& \text { Percentage crossing over }=100\left[\frac{k-8}{2 \sqrt{2(k-8)}}\right]
\end{aligned}
$$

Formulae for calculating the percentage of crossing over from observed values Coupling phase

Substituting in formula 39 , the value of $k$ obtained in formula 2, we have 
Percentage crossing over $=100\left[\frac{\left(16-\frac{16\left(A B_{0}+a b_{0}\right)}{n_{0}}\right)}{2 \sqrt{2\left(\frac{16\left(A B_{0}+a b_{0}\right)}{n_{0}}\right)-16}+8}\right]$

which is equivalent to

Percentage crossing over $=100\left[1-\sqrt{\frac{\overline{A B_{0}-A b_{0}-a B_{0}+a b_{0}}}{A B_{0}+A b_{0}+a B_{0}+a b_{0}}}\right]$

Substituting $E_{0}$ for the sum of $A B_{0}$ and $a b_{0}, M_{0}$ for the sum of $A b_{0}$ and $a B_{0}$, and $n_{0}$ for the sum of $A B_{0}, A b_{0}, a B_{0}$ and $a b_{0}$, we have,

Percentage of crossing over $=100\left[1-\sqrt{\frac{E_{0}-M_{0}}{n_{0}}}\right]$

Repulsion phase

Substituting in formula 43 , the value of $k$ obtained in formula 2 , we have

Percentage crossing over $=110\left[\frac{\frac{16\left(A B_{0}+a b_{0}\right)}{n_{0}}-8}{2 \sqrt{2\left(\frac{16\left(A B_{0}+a b_{0}\right)}{n_{0}}-8\right)}}\right]$

which is equivalent to

Percentage crossing over $=100 \sqrt{\frac{\overline{A B_{0}-A b_{0}-a B_{0}+a b_{0}}}{A B_{0}+A b_{0}+a B_{0}+a b_{0}}}$

Substituting $E_{0}$ for the sum of $A B_{0}$ and $a b_{0}, M_{0}$ for the sum of $A b_{0}$ and $a B_{0}$, and $n_{0}$ for the sum of $A B_{0}, A b_{0}, a B_{0}$ and $a b_{0}$, we have

Percentage crossing over $=100 \sqrt{\frac{\bar{E}_{0}-\bar{M}_{0}}{n_{0}}}$

The percentage of crossing over may also be obtained by dividing $E_{0}$ by $M_{0}$ and referring to table 3 . Fractional percentages can be obtained by interpolation. As an example, the percentage of crossing over may be calculated as follows from the first distribution in table 4 :

$$
\begin{aligned}
& E_{0}=631 ; \quad M_{0}=50 \\
& \frac{E_{0}}{M_{0}}=12.6
\end{aligned}
$$


By referring to table 3 we find that when the value of $E_{0} \div M_{0}=13.8038$ the percentage of crossing over is 7 and when the value of $E_{0} \div M_{0}=$ 12.0208 the percentage of crossing over is 8 . The difference $=1.7830$

$$
\begin{aligned}
& 13.8038-12.6=1.2038 \\
& \frac{1.2038}{1.7830} \text { of } 1 \text { per cent }=0.67 \text { per cent } \\
& 7.0 \text { per cent }+0.67 \text { per cent }=7.67 \text { per cent } \\
& \text { TABLE } 3
\end{aligned}
$$

\begin{tabular}{|c|c|c|c|c|c|c|c|}
\hline \multicolumn{4}{|c|}{ COUPIING PHASE } & \multicolumn{4}{|c|}{ REPULSION PHASE } \\
\hline $\begin{array}{l}\text { Percent } \\
\text { crossing } \\
\text { over }\end{array}$ & $\frac{E_{0}}{M_{0}}$ & $\begin{array}{c}\text { Percent } \\
\text { crossing } \\
\text { over }\end{array}$ & $\frac{E_{0}}{M_{0}}$ & $\begin{array}{l}\text { Percent } \\
\text { crossing } \\
\text { over }\end{array}$ & $\frac{E_{0}}{M_{0}}$ & $\begin{array}{r}\text { Percent } \\
\text { crossing } \\
\text { over }\end{array}$ & $\frac{E_{0}}{M_{0}}$ \\
\hline 1 & 99.5025 & 26 & 3.4209 & 1 & 1.0002 & 26 & 1.1450 \\
\hline 2 & 49.5051 & 27 & 3.2817 & 2 & 1.0008 & 27 & 1.1573 \\
\hline 3 & 32.8409 & 28 & 3.1528 & 3 & 1.0018 & 28 & 1.1701 \\
\hline 4 & 24.5102 & 29 & 3.0331 & 4 & 1.0032 & 29 & 1.1836 \\
\hline 5 & 19.5128 & 30 & 2.9215 & 5 & 1.0050 & 30 & 1.1978 \\
\hline 6 & 16.1822 & 31 & 2.8175 & 6 & 1.0072 & 31 & 1.2126 \\
\hline 7 & 13.8038 & 32 & 2.7202 & 7 & 1.0098 & 32 & 1.2282 \\
\hline 8 & 12.0208 & 33 & 2.6291 & 8 & 1.0129 & 33 & 1.2444 \\
\hline 9 & 10.6346 & 34 & 2.5436 & 9 & 1.0163 & 34 & 1.2614 \\
\hline 10 & 9.5263 & 35 & 2.4631 & 10 & 1.0202 & 35 & 1.2792 \\
\hline 11 & 8.6200 & 36 & 2.3875 & 11 & 1.0245 & 36 & 1.2978 \\
\hline 12 & 7.8652 & 37 & 2.3162 & 12 & 1.0292 & 37 & 1.3172 \\
\hline 13 & 7.2271 & 38 & 2.2488 & 13 & 1.0344 & 38 & 1.3375 \\
\hline 14 & 6.6805 & 39 & 2.1852 & 14 & 1.0400 & 39 & 1.3588 \\
\hline 15 & 6.2072 & 40 & 2.1250 & 15 & 1.0460 & ro & 1.3810 \\
\hline 16 & 5.7935 & 41 & 2.0679 & 16 & 1.0525 & 41 & 1.4041 \\
\hline 17 & 5.4288 & 42 & 2.0139 & 17 & 1.0595 & 42 & 1.4283 \\
\hline 18 & 5.1050 & 43 & 1.9625 & 18 & 1.0670 & 43 & 1.4537 \\
\hline 19 & 4.8157 & 44 & 1.9137 & 19 & 1.0749 & 44 & 1.4802 \\
\hline 20 & 4.5556 & 45 & 1.8674 & 20 & 1.0833 & 45 & 1.5078 \\
\hline 21 & 4.3206 & 46 & 1.8232 & 21 & 1.0923 & 46 & 1.5368 \\
\hline 22 & 4.1073 & 47 & 1.7813 & 22 & 1.1017 & 47 & 1.5670 \\
\hline 23 & 3.9128 & 48 & 1.7412 & 23 & 1.1117 & 48 & 1.5988 \\
\hline 24 & 3.7349 & 49 & 1.7031 & 24 & 1.1222 & 49 & 1.6319 \\
\hline 25 & 3.5714 & 50 & 1.6667 & 25 & 1.1333 & 50 & 1.6667 \\
\hline
\end{tabular}

Crossing-over percentages from 1 to 50 and the corresponding ratios of $\mathrm{E}_{0}$ to $\mathrm{M}_{0}$.

\section{DISCUSSION}

In order to illustrate the method of application of some of the formulae, table 4 was compiled. The data in this table are the same as those used by Emerson (1916). Formula 2 was first used to obtain the value of $k$. From the value of $k$, the value of $n$ was calculated by using formula 8 for 
the first two distributions and formula 12 for the third distribution. Finding the value of $n$ in the coupling phase involves several computations because formula 8 is quite complex, but after $n$ has once been obtained the calculation from $n$, of the other members of the zygotic series, is relatively simple. The value of $k$ in the fourth distribution is 7.94. Since this value is less than 8, the repulsion formulae are not applicable. EMERSON (1916) found that the value of $r$ for this distribution could not be determined by the formula which he proposed because $(1335+2)-(643+714)$ is a negative quantity $(-20)$ and has no real root.

TABLE 4

Theoretical values of various terms obtained from observed values.

\begin{tabular}{|c|c|c|c|c|c|c|c|c|c|c|c|}
\hline \multicolumn{5}{|c|}{ OBSERVED } & \multicolumn{7}{|c|}{ THEORETICAI } \\
\hline$A B$ & $A b_{0}$ & $a B_{0}$ & $a b o$ & $n_{v}$ & $k$ & $r: s$ & $A B$ & $A b$ & $a B$ & $a b$ & $n$ \\
\hline 493 & 25 & 25 & 138 & 681 & 14.825 & $12.1: 1$ & 488.8 & 25.2 & 25.2 & 146.1 & 685.3 \\
\hline 165 & 58 & 58 & 78 & 359 & 10.830 & $1.46: 1$ & 14.30 & 3.93 & 3.93 & 2.13 & 24.34 \\
\hline 336 & 150 & 143 & 11 & 640 & 8.675 & $1: 2.44$ & 24.7 & 10.85 & 10.85 & 1 & 47.4 \\
\hline 1335 & 643 & 714 & 2 & 2694 & 7.94 & 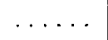 & 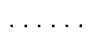 & $\ldots \ldots$ & $\ldots \ldots$ & . & $\ldots \ldots$ \\
\hline
\end{tabular}

The formulae reported in this paper are applicable only when the value of $k$ is between 10 and 8 (repulsion phase) or 10 and 16 (coupling phase). With a reduction of the same number of $A B_{0}$ or $a b_{0}$ types for a given value of $r$ or $s$ the limits for the application of the formulae are reached sooner in the repulsion phase than in the coupling phase as is indicated by the nature of the curves in figures 1 and 2 . In the coupling phase the value of the points on the curve approaches 16 gradually, while in the repulsion phase it approaches 8 rapidly at first, then more slowly.

The relationship of $E_{0}$ and $M_{0}$ to $r$ and $s$ is shown in the following formulae:

$$
\sqrt{\frac{E_{0}-M_{0}}{E_{0}+M_{0}}}=\frac{r}{r+1} \quad \text { (coupling phase) }
$$

and

$$
\sqrt{\frac{E_{0}-M_{0}}{E_{\theta}+M_{0}}}=\frac{1}{s+1} \quad \text { (repulsion phase) }
$$

The relationship between $E_{0}$ and $M_{0}$ may also be expressed as follows:

and

$$
E_{0}=M_{0}+4 c r^{2} \quad \text { (coupling phase) }
$$

$$
E_{0}=M_{0}+4 c \quad \text { (repulsion phase) }
$$




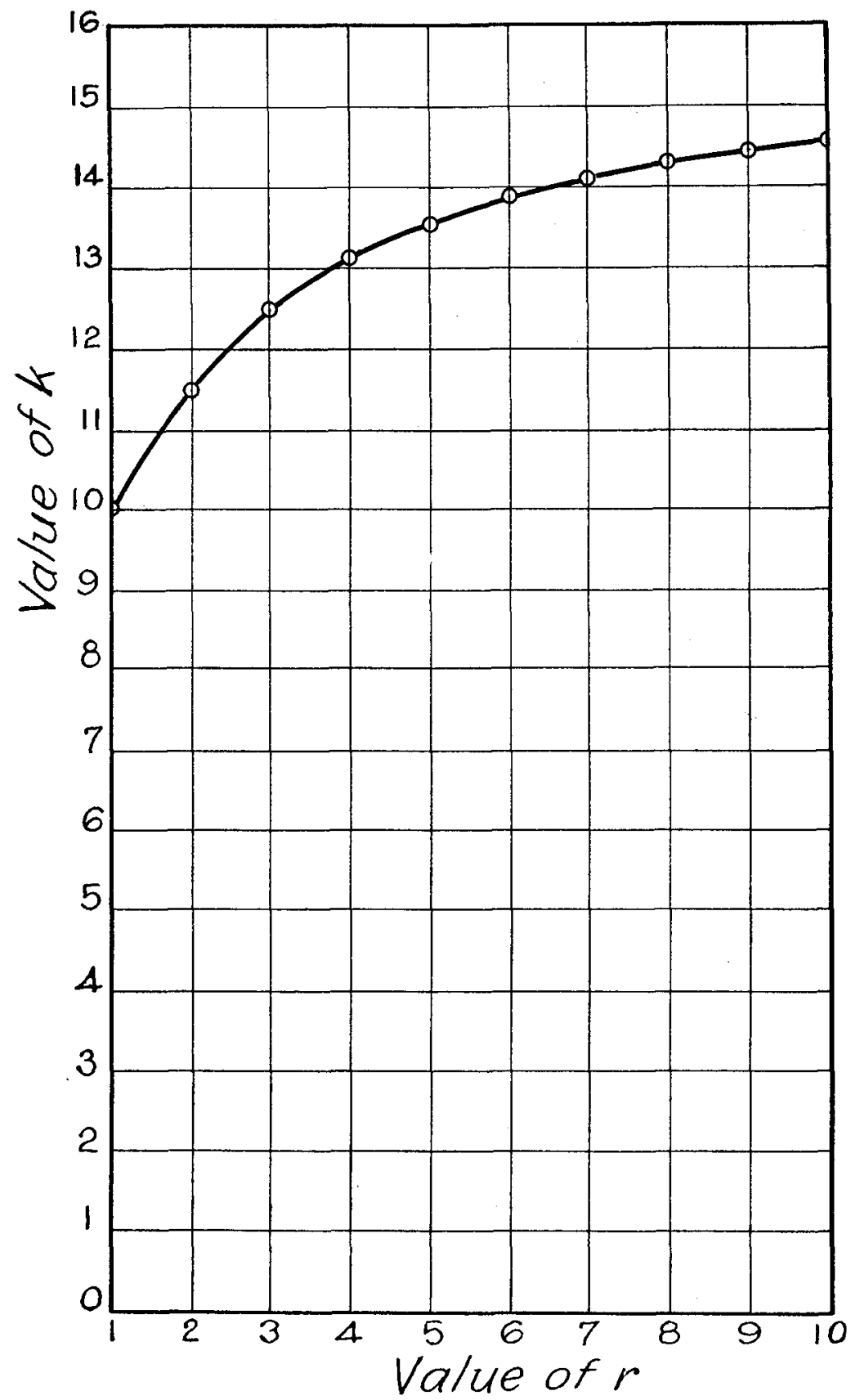

FIGURE 1.-The curve shows that when the value of $r$ is increased, the value of $k$ approaches 16 gradually. 
A further relationship between $E_{0}$ and $M_{0}$ is indicated by formulae 50 and 51:

and

$$
\frac{E_{0}}{M_{0}}=r+\frac{1}{2}+\frac{c}{M_{0}} \quad \text { (coupling phase) }
$$

$$
\frac{E_{0}}{M_{0}}=1+\frac{4 c}{M_{0}} \quad \text { (repulsion phase) }
$$

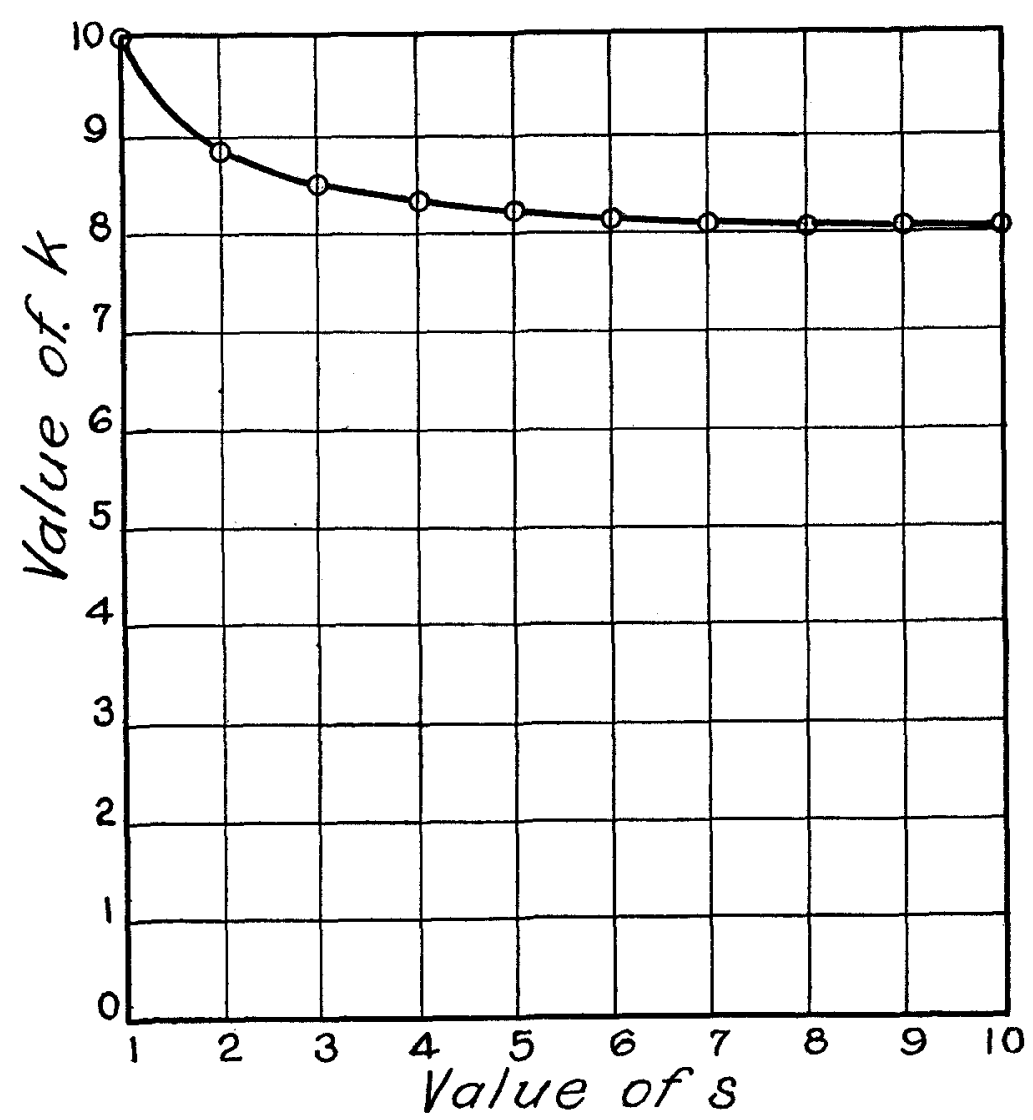

FIgURE 2.-The curve shows that when the value of $s$ is increased, the value of $k$ approaches 8 , rapidly at first, then more slowly.

The value of $E_{0}$ divided by $M_{0}$ in the coupling phase is approximately equal to $r+\frac{1}{2}$ and the greater the magnitude of $r$ the less is the magnitude of $\frac{\mathrm{c}}{\mathrm{M}_{0}}$ which approaches zero as the value of $r$ approaches infinity. On the other hand, the value of $E_{0}$ divided by $M_{0}$ in the repulsion phase is approximately equal to 1 , and the magnitude of $\frac{4 \mathrm{c}}{\mathbf{M}_{0}}$ decreases as the 
value of $s$ increases. The value of $\frac{4 c}{\mathbf{M}_{0}}$ approaches zero as the value of $s$ approaches infinity. In other words, the ratio of $E_{0}$ to $M_{0}$ is approximately equal to the magnitude of $r+\frac{1}{2}$ in the coupling phase while in the repulsion phase $E_{0}$ is approximately equal to $M_{0}$. With this relationship in mind it is evident that a considerable reduction of $A B_{0}$ and $a b_{0}$ types, caused perhaps by differential viability or selective fertilization, is permissible in the coupling phase to meet the conditions necessary for the application of the formulae. The effect of such reductions, however, is not corrected by the formulae and thus an erroneous value for $r$ is obtained. On the other hand, owing to the approximate equality of $E_{0}$ and $M_{0}$ in the repulsion phase, a slight reduction of the $A B_{0}$ and $a b_{0}$ types may cause the value of $E_{0}$ to be less than $M_{0}$. If the reduction of these types is greater than $4 c$, then $E_{0}$ minus $M_{0}$ will have a negative value.

In order that the formulae may be usable, the observed values should deviate as little as possible from the result obtained by multiplying the theoretical distribution by $c$. If the observed distribution corresponds to this distribution, the quotient obtained by dividing the sum of the types $A B_{0}$ and $A b_{0}$ by the types $a B_{0}$ and $a b_{0}$ is a constant, being equal to 3 in both coupling and repulsion phases.

$$
\frac{A B_{0}+A b_{0}}{a B_{0}+a b_{0}}=3
$$

\section{SUMMARY}

1. A constant $k$ which shows the relation of observed values in linkage studies to independent inheritance is obtained.

2. The value of $k$ indicates the following:

(a) When $k=10$, independent inheritance.

(b) When $k=$ more than 10 but less than 16 , coupling.

(c) When $k=$ less than 10 but more than 8 , repulsion.

(d) When $k=$ less than 8 , some factor other than chance resulting in marked deviations from theoretical ratios.

3. Formulae are given showing the relation of the members of the theoretical gametic and zygotic series to one another.

4. The relationship between the extreme terms and the middle terms of a distribution is shown. 
5. Formulae and tables for determining the percentage of crossing over are presented.

\section{ACKNOWLEDGMENT}

The writer is indebted to Professor C. M. Woodworth for helpful suggestions and constructive criticisms given during the preparation of this paper and the constant interest shown throughout its progress.

\section{LITERATURE CITED}

Brunson, A. M., 1924 The inheritance of a lethal pale green seedling character in maize. Memoir Cornell Univ. 72. pp. 1-22.

Coldins, G. N., 1924 Measurement of linkage values. Jour. Agric. Res. 27: 881-891.

EMERSON, R. A., 1916 The calculation of linkage intensities. Amer. Nat. 50: 411-420.

HALDANE, J. B. S., 1919 The probable errors of calculated linkage values and the most accurate method of determining gametic from certain zygotic series. Jour. Genetics 8: 291-297.

Woopworth, C. M., 1923 Calculation of linkage intensities where duplicate factors are concerned. Genetics 8: 106-115. 